\title{
Geração de metano e de créditos de carbono no tratamento de esgotos sanitários
}

\author{
Methane and carbon credit generation in sanitary wastewater \\ treatment
}

Aline Pereira Lima | Nemésio Neves Batista Salvador

Data de entrada: 27/01/2012 | Data de aprovação: 14/01/2014

DOI: http://dx.doi.org/10.4322/dae.2014.121

Resumo

O conceito dos créditos de carbono surgiu a partir da conscientização para a necessidade de reduzir as emissões de gases de efeito estufa (GEE) a fim de combater o agravamento do aquecimento global, discutido e formalizado no Protocolo de Quioto. A emissão de GEE acontece devido a diversas atividades, sendo que nas estações de tratamento de esgotos (ETEs), por exemplo, a geração do gás estufa metano $\left(\mathrm{CH}_{4}\right)$ por processos biológicos pode ser significativa.

Este trabalho apresenta uma estimativa da produção de $\mathrm{CH}_{4}$ nos processos mais usuais de tratamento biológico de esgotos no Brasil e sua representatividade em termos de créditos e valor monetário, com base nos métodos e procedimentos do IPCC - Intergovernmental Panel on Climate Change (IPCC, 2006). Os resultados mostram que o volume de $\mathrm{CH}_{4}$ emitido por processos anaeróbios, como lagoas e reatores anaeróbios é relevante. Dentre eles, verifica-se que a substituição de sistemas abertos por processos aeróbios representa o maior potencial de obtenção de créditos, podendo alcançar preços de $\mathrm{R} \$ 1.900,00 / 1000$ pessoas atendidas/ano. Essa possibilidade de obtenção de créditos de carbono, provenientes do Mecanismo de Desenvolvimento Limpo (MDL), pode contribuir para amortizar parte dos custos de implementação das estações de tratamento de esgotos, bem como estimular a sua modernização.

Palavras-chave: Créditos de carbono; metano; Processos biológicos de tratamento de esgoto sanitário.

\section{Abstract}

The concept of carbon credits came from the awareness of the need to reduce emissions of greenhouse gases (GHGs) in order to combat the worsening of global warming, discussed and formalized in the Kyoto Protocol. The GHG emission is due to several activities and in wastewater treatment plants (WTPs), for example, the generation of greenhouse gas methane $\left(\mathrm{CH}_{4}\right)$ by biological processes can be significant.

This paper presents an estimate of the production of $\mathrm{CH}_{4}$ in the most usual processes of biological treatment of wastewater in Brazil and its representation in terms of credit and monetary value, based on the methods and procedures of the IPCC - Intergovernmental Panel on Climate Change (IPCC, 2006). The results show that the amount of $\mathrm{CH}_{4}$ emitted by anaerobic processes such as anaerobic lagoons and is relevant. Among them, it appears that the replacement of open systems by aerobic processes represents the greatest potential for obtaining credits, which can reach prices of $R \$ 1900.00 / 1000$ customers/year. The possibility of obtaining carbon credits from the Clean Development Mechanism (CDM), can contribute to amortize part of the implementation costs of the sewage treatment plants and stimulate its upgrading.

Key-words: Carbon credits; methane; biological treatment process of sanitary wastewater.

*Curriculum dos autores - ver página 70 


\section{Introdução}

Diante da problemática das mudanças climáticas, discussões vêm ocorrendo em âmbito científico e político, culminando em uma série de programas e conferências, entre os quais o Protocolo de Quioto. Este criou três mecanismos para reduzir as emissões de carbono, sendo que um deles se refere diretamente aos países em desenvolvimento: o Mecanismo de Desenvolvimento Limpo (MDL). Por meio dele, cada tonelada de carbono (ou unidade de Redução Certificada de Emissões-RCE) que deixe de ser emitida em um país em desenvolvimento ou parte anfitriã (não listado no Anexo I do Protocolo de Quioto) pela adoção de novas tecnologias, poderá ser negociada com os países industrializados (integrantes do Anexo I) e usada como forma de cumprirem suas metas de redução de emissões, determinadas em Quioto (FGV, 2002). Este mecanismo está ilustrado na Figura 1.

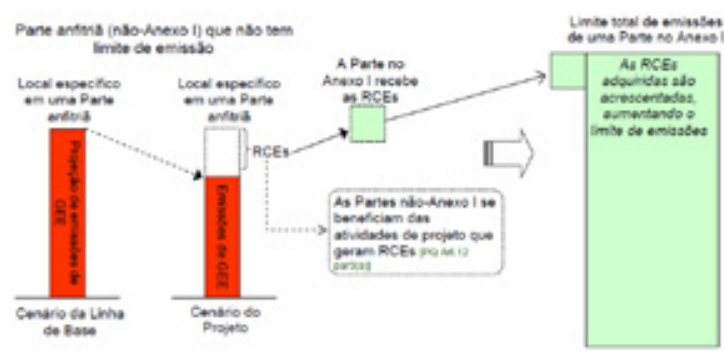

Fonte: MCT (2009b).

Figura 1 - Princípios do MDL para obtenção de RCEs

Como mostra a Figura 1, a RCE pode ser gerada a partir da diferença de emissões entre um cenário inicial (Linha de Base) e o cenário de atividade com o projeto já implementado. Não obstante, o MDL foi criado com o objetivo de fomentar o desenvolvimento sustentável mediante transferência de tecnologia e incentivo a um novo padrão de desenvolvimento com base na conciliação entre crescimento econômico, inclusão social e respeito ao meio ambiente.

É importante destacar que o aquecimento global é um fenômeno natural, causado pela concentração de gases do efeito estufa na atmosfera da Terra. Esses gases são produzidos também pela queima de combustíveis fósseis em usinas termoelétricas, indústrias, veículos, atividades agro-pastoris, tratamento e disposição de resíduos. Este incremento de origem antrópica perturba o balanço natural dos gases e têm agravado o efeito estufa (IPCC, 1996).
Nesse sentido, os gases estufa dióxido de carbono $\left(\mathrm{CO}_{2}\right)$, metano $\left(\mathrm{CH}_{4}\right)$ e óxido nitroso $\left(\mathrm{N}_{2} \mathrm{O}\right)$ são os mais emitidos por atividades antrópicas atualmente em termos de volume: $77 \%, 14 \%$ e $8 \%$, respectivamente (IPCC, 2007). Suas principais fontes globais são as atividades relacionadas à geração de energia e ao transporte - $26 \%$ e 13\% do total de emissões respectivamente, ao passo que a contribuição do setor de resíduos (que contempla o tratamento de esgoto) é de 3\% (IPCC, 2007).

A fonte de geração de GEE explorada neste trabalho é o tratamento de esgoto, cujo objetivo é remover substâncias indesejáveis ou transformá-las em outras de forma aceitável. Metcalf \& Eddy (2003) define que os métodos de tratamento nos quais há predominância de forças físicas são conhecidos como operações unitárias; enquanto que os métodos em que a remoção de contaminantes é provocada por reações químicas ou biológicas são denominados processos unitários. Assim, todos eles buscam degradar matéria orgânica e remover DBO-Demanda Bioquímica de Oxigênio e DQO-Demanda Química de Oxigênio. A remoção de poluentes visa adequar o lançamento a uma qualidade desejada ou a um padrão de qualidade estabelecido por legislação e está relacionada aos conceitos de nível e eficiência do tratamento (VON SPERLING, 1996). Os processos de tratamento mais empregados no Brasil e que são possíveis emissores de gases são mostrados na Figura 2 nos itens em destaque.

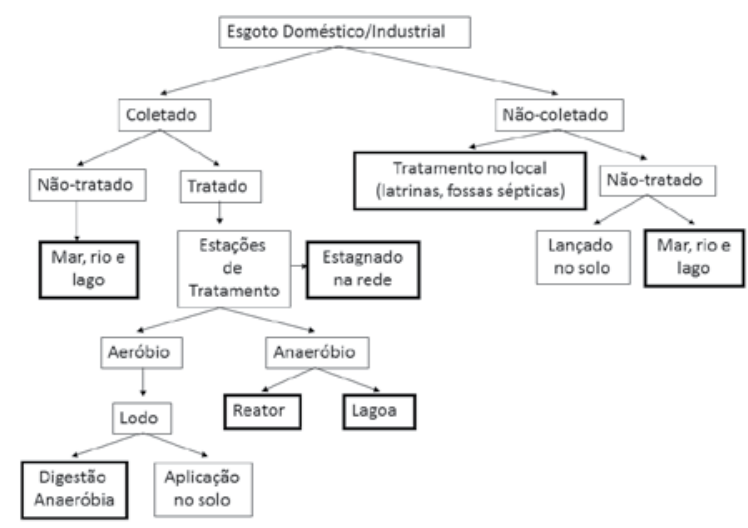

Fonte: MCT (2009b).

Figura 2 - Fluxos de esgoto, sistemas de tratamento com emissões potenciais de CH4. Fonte: IPCC (2000).

A Figura 2 destaca os métodos biológicos: reatores anaeróbios, lagoas anaeróbias e sistemas sépticos, além de lançamento em corpos d'água que 
caracteriza destinação final. Segundo Von Sperling (1996), o tratamento biológico de esgotos é uma imitação de processos que ocorrem normalmente na natureza, conhecidos como autodepuração. Portanto, o tratamento biológico de esgotos resume-se a atividade de microrganismos, principalmente bactérias, que se alimentam da matéria orgânica dos próprios resíduos. Nesse processo metabólico, diversos produtos são gerados, dentre eles, os GEE.

Essa decomposição pode ocorrer por meio de três rotas: aeróbia, anóxica e anaeróbia (ilustradas na Figura 3).

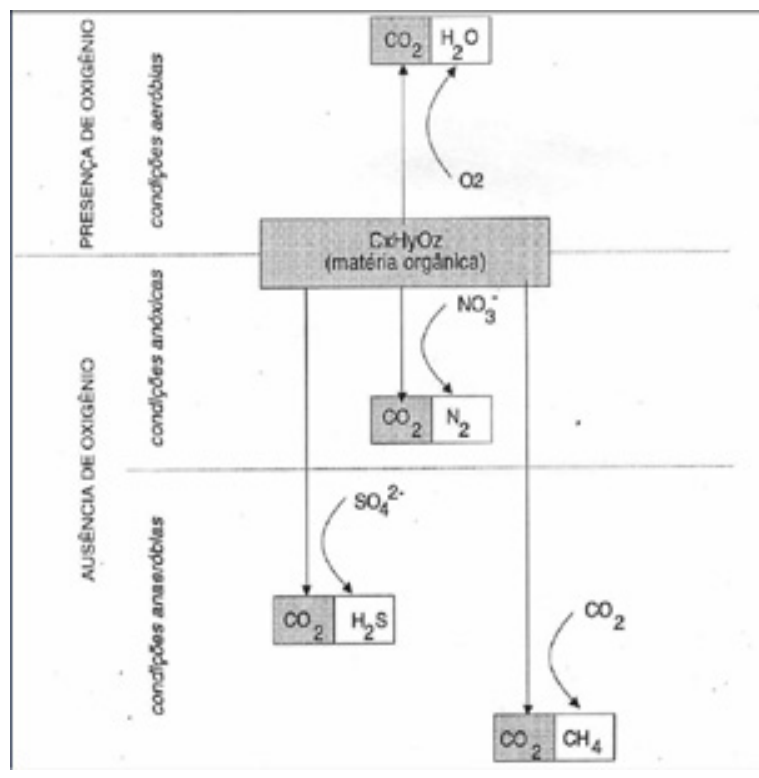

Fonte: Lubberding (1995) citado em Von Sperling (1996).

Figura 3 - Principais rotas de decomposição da matéria orgânica na presença de diferentes aceptores de elétrons.

Nesse sentido, as principais equações para a geração de energia que ocorrem em condições aeróbias, anóxicas e anaeróbias são:

Condições aeróbias:

Equação 1:

$$
\mathrm{C}_{6} \mathrm{H}_{12} \mathrm{O}_{6}+6 \mathrm{O}_{2} \rightarrow 6 \mathrm{CO}_{2}+6 \mathrm{H}_{2} \mathrm{O}
$$

Condições anóxicas - redução de nitratos (desnitrificação)

Ocorrem duas reações, envolvendo a matéria orgânica:

Equação 2:
$4 \mathrm{NO}_{3}+5 \mathrm{CH}_{2} \mathrm{O} \rightarrow 2 \mathrm{~N}_{2}+3 \mathrm{H}_{2} \mathrm{O}+5 \mathrm{CO}_{2}+4 \mathrm{OH}^{-}$

Equação 3 (formação do GEE $\mathrm{N}_{2} \mathrm{O}$ ):

$4 \mathrm{NO}_{3}{ }^{-}+4 \mathrm{CH}_{2} \mathrm{O} \rightarrow 2 \mathrm{~N}_{2} \mathrm{O}+2 \mathrm{H}_{2} \mathrm{O}+4 \mathrm{CO}_{2}+4 \mathrm{OH}^{-}$

Condições anaeróbias - redução dos sulfatos (dessulfatação)

Equação 4:

$\mathrm{CH}_{3} \mathrm{COOH}+\mathrm{SO}_{4}{ }^{2-}+2 \mathrm{H}^{+} \rightarrow \mathrm{H}_{2} \mathrm{~S}+2 \mathrm{H}_{2} \mathrm{O}+2 \mathrm{CO}_{2}$

Condições anaeróbias: redução de $\mathrm{CO}_{2}$ (metanogênese hidrogenotrófica)

Equação 5:

$$
4 \mathrm{H}_{2}+\mathrm{CO}_{2} \rightarrow \mathrm{CH}_{4}+2 \mathrm{H}_{2} \mathrm{O}
$$

Condições anaeróbias (metanogênese acetotrófica)

Equação 6:

$$
\mathrm{CH}_{3} \mathrm{COOH} \rightarrow \mathrm{CH}_{4}+\mathrm{CO}_{2}
$$

Como visto nas reações anteriores, o tratamento de esgotos sanitários emite, em termos de GEE, principalmente $\mathrm{CO}_{2}, \mathrm{CH}_{4}$ e $\mathrm{N}_{2} \mathrm{O}$. No que se refere à emissão de $\mathrm{CO}_{2}$, esta pode ser avaliada com base na demanda de energia de uma estação de tratamento de esgotos (IPCC, 2001). Entretanto, neste caso, este gás é definido como biogênico e em tratamento de esgotos não é considerado contribuinte para o efeito estufa, pois não altera desta forma o balanço dos GEEs na atmosfera. Consequentemente, as emissões de $\mathrm{CO}_{2}$ provenientes de ETEs não são passíveis de gerar créditos de carbono e, portanto, não fazem parte do escopo do presente trabalho. 
A formação do $\mathrm{CH}_{4}$ é proveniente de processos de tratamento anaeróbios, nos quais a matéria orgânica é degradada em quatro etapas: hidrólise, acidogênese, acetogênese e metanogêse. A separação da fração orgânica do esgoto (sob condições anaeróbias) acontece sequencialmente de um composto complexo para um simples, começando com a hidrólise de materiais particulados complexos para polímeros simples como proteínas, carboidratos e lipídeos. Estes são ainda novamente hidrolisados para produzir aminoácidos, açúcares, e ácidos graxos de alto peso molecular. Aminoácidos e açúcares são convertidos também para um subproduto intermediário (por exemplo, ácido propiônico, butírico e outros ácidos voláteis) ou diretamente fermentados para ácido acético. A produção de metano e dióxido de carbono ocorre por meio da clivagem do acetato (CHERNICHARO, 2005).

Já as emissões de óxido nitroso podem ocorrer na forma direta, em estações de tratamento, ou indireta, em águas residuárias lançadas nos corpos d'água. Emissões diretas da nitrificação (via aeróbia) e desnitrificação (via anóxica) em ETEs são consideradas como uma fonte minoritária se comparadas com as indiretas e podem ser apenas de interesse de países que têm avançadas estações de tratamento de esgotos, em grau terciário (IPCC, 2006). Por esta razão, o Guidelines for National Greenhouse Gas Inventories (IPCC, 2006) assume que a maior emissão do nitrogênio no setor de esgotos ocorre pela nitrificação e desnitrificação em estuários e rios, ou seja, assume que em geral não há remoção significativa de nitrogênio durante o tratamento secundário em si. Desta maneira, as emissões de $\mathrm{N}_{2} \mathrm{O}$ não são contempladas por este trabalho, pois o lançamento de águas residuárias em corpos d'água é uma forma de disposição final e não um processo de tratamento propriamente dito.

Segundo o Ministério da Ciência e Tecnologia (MCT) (2009a), o tratamento de resíduos era em 2005 responsável por $11 \%\left(2113 \mathrm{GgCH}_{4}\right)$ da emissão total anual de metano no Brasil $\left(18971 \mathrm{GgCH}_{4}\right)$, enquanto que a contribuição do tratamento de esgotos sanitários era de 2,1\% (393 $\left.\mathrm{GgCH}_{4}\right)$. Não obstante, no cenário brasileiro, a deficiência no tratamento de esgotos sanitários é um problema que atinge várias áreas: apenas 34,6\% do total de esgotos gerados são tratados (MC, 2010). Este setor constitui-se em um instrumento fundamental de promoção do desenvolvimento sustentável nacional e local, com ganhos significativos para a qualidade de vida da população, bem como para a diminuição de despesas e aplicação de recursos financeiros nos sistemas de saúde. Assim, os créditos resultantes de projetos de MDL podem estimular maiores investimentos, buscando-se a universalização do saneamento básico brasileiro. Nesse sentido, o MDL vem ao encontro dessa demanda, de modo a incentivar a instalação e modernização de ETEs, por meio da aquisição de tecnologias mais produtivas e limpas.

Dentro desse contexto, as alternativas tecnológicas para reduzir as emissões de gases estufa no tratamento de esgotos se resumem a três medidas (IPCC, 2006):

A) Substituição de um processo anaeróbio por um aeróbio. Desta forma, o tratamento passa a ter zero de emissões, se for operado adequadamente.

B) Introdução de tecnologias de recuperação e queima de metano, como flares, em sistemas existentes de tratamento anaeróbio. Neste caso, o sistema gera dióxido de carbono em vez de metano, e como já dito, o $\mathrm{CO}_{2}$ não é contabilizado. Deve-se atentar para a eficiência do queimador, que define qual a parcela do gás sofrerá combustão completa até o produto final $\left(\mathrm{CO}_{2}\right)$.

C) Introdução de tecnologias de recuperação e queima do biogás para fins energéticos. Esta alternativa tem a peculiaridade de criar uma fonte de energia a partir do biogás, por isso, a obtenção de créditos está atrelada aos requisitos e normas de projeto do setor Energia, e não mais de Resíduos como nas anteriores; exceto se esta for utilizada no tratamento do lodo, situação não contemplada neste trabalho.

Os relatórios e inventários nacionais e regionais têm apontado os volumes das emissões de metano provenientes do tratamento de esgotos sanitários que são elegíveis como projetos de MDL. Estes volumes indicam que existe potencial de redução de geração de GEE. As reduções, por sua vez, são obtidas por meio de alternativas de mitigação, trabalhadas por meio de projetos de MDL, que possuem metodologias de elaboração e desenvolvimento específicas. Há também um ciclo de etapas para a aprovação de projetos e obtenção das RCEs; mais informações a respeito podem ser encontradas no endereço eletrônico do Ministério da Ciência e Tecnologia. Entretanto, na prática, existem apenas quatro projetos de MDL brasileiros registrados no IPCC aplicados a esgotos industriais - todos com a metodologia AMS - III.I (IPCC, 1996) e não há nenhum projeto registrado envolvendo esgotos sanitários ou domésticos, também considerando seu desenvolvimento em território nacional. A partir dessa constatação, os 
principais processos de tratamento de esgotos sanitários empregados no Brasil foram estudados em termos de emissão de GEE e suas respectivas alternativas de mitigação, a fim de verificar seu potencial de geração de créditos de carbono.

\section{Experiências Internacionais}

O cenário internacional já apresenta estudos envolvendo quantificação de GEE em processos de tratamento de esgotos sanitários. Alguns deles são resumidos a seguir.

Fadel e Massoud (2001) apresentaram métodos de estimativa usados para determinar a emissão de metano de águas residuárias tendo como exemplo o caso da gestão de efluentes do Líbano. As emissões de $\mathrm{CH}_{4}$ do Líbano foram estimadas por meio da metodologia do IPCC (IPCC, 1996, já mencionada neste trabalho) e então comparadas com os resultados obtidos com outros métodos teóricos e experimentais da USEPA, Droste, Orlich e Topark. A metodologia do IPCC pareceu subestimar os resultados do tratamento dos esgotos municipais, o que pode ter acontecido devido a esta usar o valor de DBO para representar a fração orgânica enquanto que outros métodos usam a DQO. A respeito do tratamento de efluentes industriais, os resultados mantiveram-se dentro do intervalo obtido através de outros métodos.

Cakira e Stenstrom (2005) estudaram a emissão de GEE em processos aeróbios e anaeróbios de tratamento de esgotos. As análises mostraram que para esgotos com baixa carga (menor que 300 $\mathrm{mg} / \mathrm{L} \mathrm{DBO}$ ) o processo aeróbio emite menor volume de GEE. Caso contrário (altas cargas de DBO) o tratamento anaeróbio é indicado e, o ponto chave depende da eficiência relativa do processo aeróbio. A respeito do $\mathrm{CH}_{4}$ dissolvido, a recomendação dos autores é usar o tratamento anaeróbio e em seguida um aeróbio, onde ocorrerá a oxidação biológica dessa fração do metano, pois a redução líquida de gases depende dessa reação de oxidação adicional.

Rosso e Stenstrom (2008) analisaram o potencial de sequestro de carbono no tratamento de esgotos municipais. Eles afirmaram que o tratamento adequado dos esgotos municipais pode ser considerado um fator de mitigação do aquecimento global. A análise de dados projetou benefícios para áreas urbanas sem tratamento sanitário e quantificou os atuais benefícios para os locais já abastecidos. Mostrou ainda que a produção mundial líquida de $\mathrm{CO}_{2}$ pode alcançar $191 \mathrm{ktCO}_{2}$ /dia em 2025 resultante da ausência de tratamento nas cidades. Assim, o estudo apontou um grande potencial de expansão do serviço de tratamento bem como de obtenção de créditos de carbono.

Shahabadi et al. (2010), por sua vez, desenvolveram um modelo matemático que possibilita estimar a geração de GEE por um sistema de tratamento de esgoto e determinar a contribuição de processos individuais para emissões on-site e off-site. Ele aponta ainda, entre outros, que a emissão de GEE deverá tornar-se o fator de maior influência na escolha dos processos de tratamento.

Lucas et al. (2007) buscaram a literatura existente para avaliar o potencial de redução a longo prazo dos diferentes GEE não- $\mathrm{CO}_{2}$ e apresentaram uma metodologia para estender as curvas de projeção de curto prazo para o ano de 2100. A metodologia escolhida utiliza um fator de desenvolvimento tecnológico e amplia ainda mais as curvas usando o potencial máximo de redução e o acompanhamento de custos.

Kampschreur et al. (2009) apresentaram uma revisão bibliográfica a fim de identificar os processos biológicos e as condições-chave dos processos responsáveis pela emissão de $\mathrm{N}_{2} \mathrm{O}$. A análise da literatura permitiu a identificação dos parâmetros operacionais mais importantes líderes na emissão de $\mathrm{N}_{2} \mathrm{O}$ em ETEs: (i) baixa concentração de oxigênio dissolvido (OD) nas etapas de nitrificação e desnitrificação, (ii) aumento das concentrações de nitrito em ambas as etapas de nitrificação e desnitrificação e, (iii) baixa taxa de DQO/N na etapa de desnitrificação. Segundo Kampschreur et al. (2009), a literatura não está clara sobre se os microrganismos da nitrificação e desnitrificação são a principal fonte de emissão de $\mathrm{N}_{2} \mathrm{O}$. Estratégias operacionais para evitar as emissões de $\mathrm{N}_{2} \mathrm{O}$ de ETEs são discutidas e as áreas que necessitam de pesquisa são identificadas.

\section{Procedimentos metodológicos}

A seguir são apresentados os procedimentos metodológicos para estimar a produção desses gases no setor utilizando como base o método do IPCC (IPCC, 2006) e as recomendações da United States Environmental Protection Agency (USEPA, 2010). Este método foi o adotado, pois permite que os países adaptem a abordagem da estimativa mais precisamente para seus processos de tratamento. Isto é possível devido à variável Fator de Conversão do Metano (Methane Conversion Factor - MCF), que representa o potencial de geração de $\mathrm{CH}_{4}$ em diferentes sistemas de manejo de águas residuárias. Além disso, o método 
faz uso de variáveis que são facilmente encontradas e disponíveis na maioria dos países.

As emissões de $\mathrm{CH}_{4}$ do tratamento dos esgotos sanitários foram estimadas por meio das Equações 7, 8, 910 seguintes, cujas variáveis e valores adotados estão na Tabela 1 .

Equação 7: Emissões de metano pelo tratamento de efluentes sanitários.

$$
\mathrm{CH}_{4} \text { Emissions }=\left(\mathrm{TOW}_{\text {dom }} \times \mathrm{EF}\right)-\mathrm{R}
$$

Equação 8: Estimativa do efluente doméstico orgânico total.

$$
T O W_{\text {dom }}=P o p_{\text {urb }} \times D_{\text {dom }}
$$

Equação 9: Fator de emissão.

$$
E F=B_{o} \times \text { MédiaponderadadosMCF }
$$

Equação 10: Média ponderada dos MCFi.

$$
M C F_{i}=\sum_{x}\left(W S_{i, x} x M C F_{x}\right)
$$

A seguir, efetuou-se a conversão para $\mathrm{tCO}_{2} \mathrm{e}$ dos resultados anteriores baseado no Potencial para Aquecimento Global - PAG do gás metano. Isto permitiu uniformizar os dados, para sua comparação, discussão e soma, e indicar o total de $\mathrm{CO}_{2}$ equivalente produzido pelos processos de tratamento de esgoto.

Assim, a Equação 11 foi utilizada para estimar as emissões de GEE de cada um dos processos de tratamento de esgotos sanitários em termos de $\mathrm{tCO}_{2} \mathrm{e}$, para uma comunidade de 1000 habitantes, tomada como exemplo.

Equação (11): Emissões de CO2e do tratamento de esgotos sanitários.

$$
\begin{aligned}
& \mathrm{CO}_{2} \text { eEmissions }=\left(21 \mathrm{xCH}_{4} \text { Emissions }\right) \\
& +\left(310 \mathrm{xN}_{2} \mathrm{OEmissions}\right)
\end{aligned}
$$

\begin{tabular}{|c|c|c|c|}
\hline VARIÁVEL & DESCRIÇÃO & VALOR & UNIDADE \\
\hline $\mathrm{CH}_{4}$ Emissions & Quantidade de metano gerada ao ano & a calcular & $\mathrm{tCH}_{4}$ /ano \\
\hline TOW dom & Efluente doméstico orgânico total & a calcular & $\mathrm{kgDBO} / \mathrm{ano}$ \\
\hline$E F$ & Fator de emissão & a calcular & $\mathrm{kgCH}_{4} / \mathrm{kgDBO}$ \\
\hline$R$ & $\mathrm{CH}_{4}$ recuperado ao ano & a calcular & $\mathrm{kgCH}_{4} / \mathrm{ano}$ \\
\hline Pop $_{\text {urb }}$ & População urbana atendida & 1000 & pessoas \\
\hline$D_{\text {dom }}$ & $\begin{array}{l}\text { Componente orgânico degradável do } \\
\text { efluente doméstico }\end{array}$ & $\begin{array}{c}19,71 \\
\text { NBR12209/92 (ABNT, 1992) }\end{array}$ & kgDBO/1000pessoas/ano \\
\hline$B_{o}$ & $\begin{array}{l}\text { Capacidade máxima de produção de } \\
\text { metano }\end{array}$ & 0,60 (default IPCC) & $\mathrm{kgCH}_{4} / \mathrm{kgDBO}$ \\
\hline$W S_{i, x}$ & $\begin{array}{c}\text { Fração do efluente do tipo "i" tratada } \\
\text { usando o processo " } x \text { " }\end{array}$ & a calcular & adimensional \\
\hline$M C F_{i}$ & $\begin{array}{l}\text { Fator de conversão de metano do pro- } \\
\text { cesso " } x \text { " tratando o efluente "i" }\end{array}$ & Tabela 2 & adimensional \\
\hline
\end{tabular}

O tipo de processo " $\mathrm{x}$ " e o arranjo de tratamento foram selecionados a partir dos trabalhos da CETESB (2010) e de Salvador (2010).

Fonte: Adaptado de CETESB (2010) e IPCC (2006).

Tabela 1. Variáveis para quantificação das emissões de metano. 
Neste sentido, o valor $R$ foi considerado apenas para reatores anaeróbios e digestores anaeróbios de lodos ativados, que contêm sempre um queimador, pois essa é a prática verificada no Brasil de acordo a CETESB (2010). Dessa forma, adotou-se que 100\% do metano dessas instalações é recuperado e queimado por flares, cuja eficiência é de 50\%. Além disso, foi adotado o valor 21 (adimensional) para o PAG do metano, e para os fatores de conversão de metano de cada um dos processos tratando efluente serão usados os default do IPCC (2006), mostrados na Tabela 2.

Por fim, realizou-se a conversão das RCEs para o valor monetário através da multiplicação do preço unitário da tonelada de carbono pelo total de emissões de cada processo. Adotou-se a cotação de carbono $€ 4,19 / \mathrm{tCO}_{2} \mathrm{e}$ e o valor do Euro de $\mathrm{R} \$ 2,35$, divulgados respectivamente pela Bolsa do Clima Européia ou ECX - European Climate Exchange (https:// www.theice.com/productguide/ProductGroupHierarchy.shtml?groupDetail=\&group.groupId=19) e BM\&FBOVESPA - Bolsa de Valores de São Paulo e Bolsa de Mercadorias \& Futuros (http://www.bmfbovespa.com.br), relativos ao dia 5 de janeiro de 2012 .
Equação 12: Valor monetário relativo às emissões de $\mathrm{CO}_{2}$ e de cada processo.

$$
\text { Valor }=\mathrm{CO}_{2} \mathrm{eEmissions} \times 2,35 \times 4,19
$$

\section{Resultados e discussão}

Os resultados das emissões podem ser vistos na Tabela 2.

A partir dos dados da Tabela 2 pode-se notar que a lagoa anaeróbia, a fossa séptica, os reatores anaeróbios e os digestores anaeróbios de lodo (do sistema de lodos ativados) foram os líderes em termos de emissões de metano, alcançando valores superiores a quatro toneladas por mil pessoas por ano. Isto possivelmente devido à condição na qual esses processos ocorrem: anaerobiose.

A produção de metano na fossa séptica foi um pouco menor que a das lagoas anaeróbias provavelmente devido à ocorrência predominante do fenômeno da decantação (ação física) no efluente tratado pela fossa. Sendo os tanques sépticos, reatores de fluxo horizontal, e tendo lodo passivo em relação à fase líquida, o processo biológico

\begin{tabular}{c|c|c|} 
PROCESSO & $\mathrm{MCF}_{\mathrm{i}}$ & $\mathrm{EmissõesCH}_{4}$ \\
\hline Unidade de medida & adimensional & $\begin{array}{c}\mathrm{tCH}_{4} / \\
\text { pessoas/ano }\end{array}$ \\
\hline Tanque Séptico (TS) & 0,5 & 5,9 \\
\hline Reator Anaeróbio de manta de lodo e fluxo ascendente (RAFA) & 0,8 & 4,7 \\
\hline Reator Anaeróbio Compartimentado (RAC) & 0,8 & 4,7 \\
\hline Lagoa Anaeróbia (profundidade maior que 3 metros) (Lan) & 0,8 & 9,5 \\
\hline Lagoa Facultativa (profundidade menor que 2 metros) (Lfac) & 0,2 & 2,4 \\
\hline Lagoa Aerada Facultativa (profundidade maior que 2 metros) (LaerF) & 0,2 & 2,4 \\
\hline Lagoa Aerada (profundidade maior que 2 metros) (LAer) & 0,1 & 1,2 \\
\hline Lodos Ativados Convencional (Digestor de Lodo) (LAC(DL)) & 0,8 & 4,7 \\
\hline Lodos Ativados Convencional (Tanque de Aeração sobrecarregado) (LAC(TA)) & 0,3 & 3,5 \\
\hline Lodos Ativados por Aeração Prolongada (Digestor de Lodo) (LAAP(DL)) & 0,8 & 4,7 \\
\hline Lodos Ativados por Aeração Prolongada (Tanque de Aeração sobrecarregado) & 0,3 & 3,5 \\
\hline (LAAP(TA)) & 0,8 & 4,7 \\
\hline Lodos Ativados por Batelada (Tanque de Aeração sobrecarregado) (LAB (TA)) & 0,3 & 3,5 \\
\hline
\end{tabular}

Tabela 2. Valores de MCF e estimativa da produção de metano. 
que ocorre no esgoto é menos significativo, consequentemente, a produção de gases é menor. Entretanto, destaca-se que o fato de a emissão dos digestores e reatores (anaeróbios) ser menor entre os processos anaeróbios está relacionado à existência de um queimador, que captura parte desse gás (metano recuperado- $R$ ), prática verificada no Brasil.

Quanto à produção de metano no tanque de aeração de lodos ativados, considerou-se para a estimativa, um cenário no qual o sistema é mal operado e/ou a ETE encontra-se sobrecarregada. Desta forma, o oxigênio foi consumido totalmente no processo aeróbio, criando condições anaeróbias no tanque e produzindo $3,5 \mathrm{tCH}_{4} / 1000$ pessoas/ano ou quase $75 \mathrm{tCO}_{2} \mathrm{e} / 1000$ pessoas/ano como exibido na Figura 4.

Afigura 4 mostraqueaslagoasaeradassãoasmenores emissoras de GEE $\left(1,2 \mathrm{tCH}_{4} / 1000\right.$ pessoas/ ano ou $25 \mathrm{tCO}_{2} \mathrm{e} / 1000$ pessoas/ano), seguidas das lagoas facultativas $\left(2,4 \mathrm{tCH}_{4} / 1000\right.$ pessoas/ ano ou quase $50 \mathrm{tCO}_{2} \mathrm{e} / 1000$ pessoas/ano), mesmo sem existir qualquer tipo de captura de gases associado a esses sistemas.

As emissões de metano das lagoas anaeróbias $\left(\mathrm{em} \mathrm{CO}_{2} \mathrm{e}\right)$ foram elevadas em relação a contribuição dos demais processos de lagoas de estabilização. Entretanto, deve-se considerar o uso de associações desses processos.

Um aspecto relevante é o fato de que as emissões do tanque de aeração e do digestor de lodo do sistema de lodos ativados são tratadas separadamente. Assim, o arranjo usual lodo ativado e digestor deve produzir um volume de gás diferente da soma das parcelas calculadas neste trabalho. Isto porque as unidades funcionam sequencialmente, de maneira a mudar a carga orgânica afluente no digestor de lodo. Portanto, recomenda-se o estudo das emissões para os principais arranjos de processos de tratamento usualmente empregados.

A partir do total de toneladas de carbono equivalente emitidas, escolheu-se para cada

\section{Emissões de CO2e (tCO2e/1000pessoas/ano)}

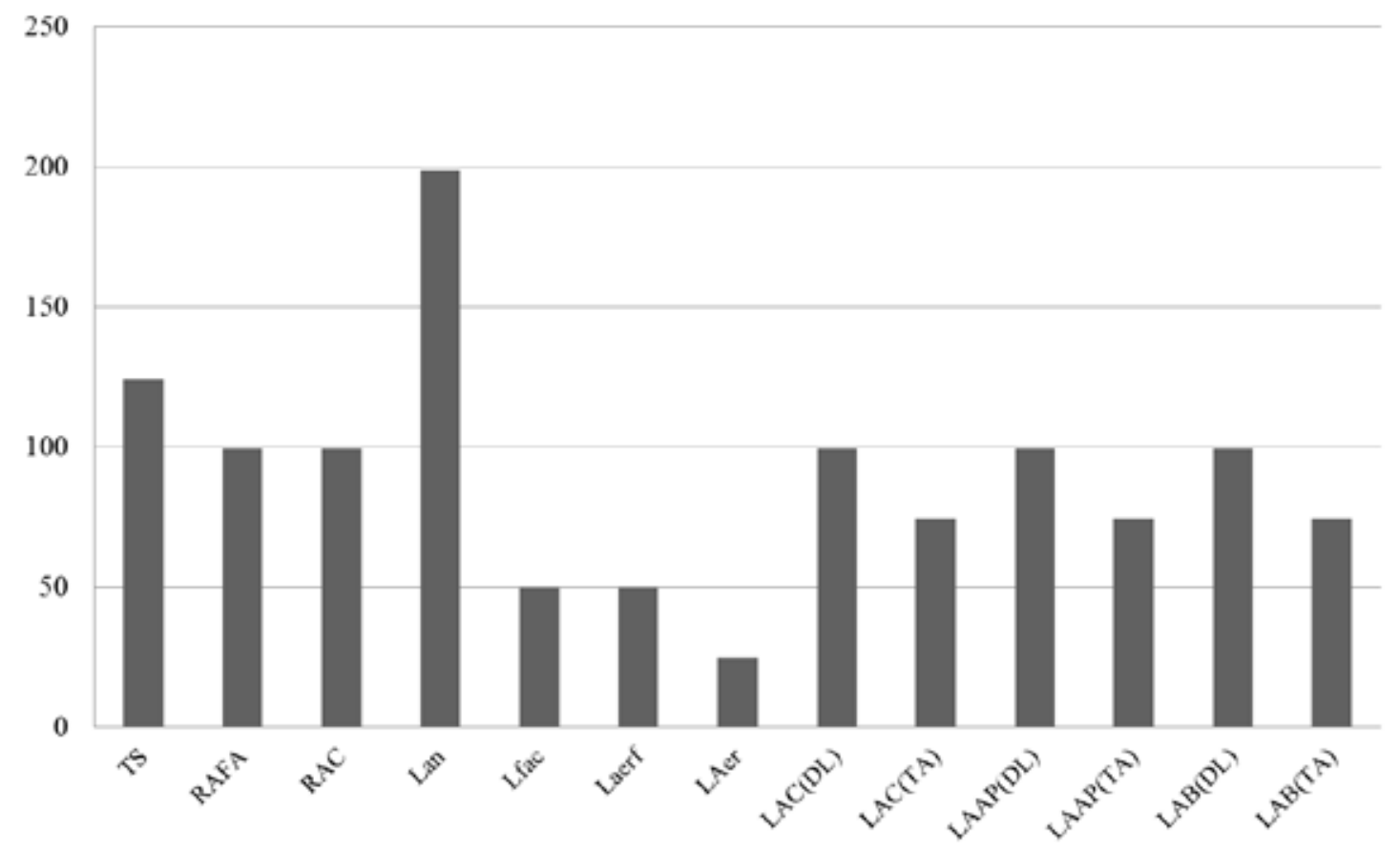

Figura 4 - Emissões de $\mathrm{CO}_{2}$ e ( $\mathrm{tCO}_{2} \mathrm{e} / 1000$ pessoas/ano) nos processos de tratamento de esgoto. 
processo uma alternativa tecnológica e calculou-se o número de RCEs (toneladas que deixam de ser geradas) e seu respectivo valor em dinheiro (Tabela 3). É relevante lembrar que se trata de um cálculo simplificado, pois existem metodologias e exigências específicas (ciclo de projeto) para cada caso de substituição ou adição de tecnologia. Optou-se neste trabalho por uma abordagem geral a fim de mostrar o potencial do setor de tratamento de esgoto.

Para o cálculo de RCEs geradas, sabendo que cada tonelada de carbono corresponde a um certificado, adotou-se o volume de emissões em números inteiros, e sobre eles calculou-se o valor em dinheiro baseado na cotação do carbono.

De acordo com a Tabela 3, quatro processos foram escolhidos para serem substituídos por métodos aeróbios (alternativa "A"): tanque séptico (TS), lagoa anaeróbia (Lan), lagoa facultativa (Lfac) e lagoa aerada facultativa (Laerf). Isto porque, estes são sistemas abertos que não permitem recuperação de gás. Assim, os projetos reduziriam suas emissões a zero e conseguiriam, cada um, preços entre $\mathrm{R} \$$ 500/1000pessoas/ano no caso das fossas sépticas e R \$1950 por milhar de pessoas atendidas ao ano para lagoas anaeróbias.

Aos reatores e digestores de lodo anaeróbios (RAFA, LAC(DL), LAAP(DL) e LAB(DL)), a sugestão seria a introdução de queimadores (alternativa de mitigação "B”). Esta prática já é verificada com frequência no Brasil com uso de equipamentos com eficiência de 50\%. Portanto, para este cenário, recomendou-se melhorar a eficiência para a máxima (próxima a $100 \%$ produção de $\mathrm{CO}_{2}$. Porém, dado que o MDL é um instrumento adicional e o Protocolo de Quioto prioriza medidas internas de redução de emissão de GEE, esta medida tem poucas possibilidades de gerar créditos. Consequentemente o cálculo da geração de RCEs e seu valor monetário não foi aplicado.

Os demais processos (Lagoa Aerada - Laer e os tanques de aeração das três variações de Lodos Ativados - LAC(TA), LAAP(TA) e LAB(TA)) são aeróbios, entretanto, sob más condições de funcionamento e manutenção podem gerar GEE.

\begin{tabular}{|c|c|c|c|}
\hline PROCESSO & $\begin{array}{c}\text { Alternativa de } \\
\text { mitigação }\end{array}$ & RCEs geradas & Valor monetário \\
\hline Unidade de medida & & & R\$/1000pessoas/ano \\
\hline TS & A & 124 & 1220,966 \\
\hline RAFA & B & - & - \\
\hline RAC & B & - & - \\
\hline Lan (profundidade maior que 3 metros) & A & 198 & 1949,607 \\
\hline Lfac (profundidade menor que 2 metros) & A & 49 & 482,4785 \\
\hline Laerf (profundidade maior que 2 metros) & A & 49 & 482,4785 \\
\hline Laer (profundidade maior que 2 metros) & $*$ & - & - \\
\hline LAC(DL) & B & - & - \\
\hline LAC(TA) & $*$ & - & - \\
\hline LAAP(DL) & B & - & - \\
\hline LAAP(TA) & $*$ & - & - \\
\hline LAB(DL) & $*$ & - & - \\
\hline LAB(TA) & $*$ & - & - \\
\hline
\end{tabular}

As letras "A", "B" são relativas às medidas de mitigação expostas na seção de Revisão Bibliográfica. A alternativa "C" não entrou no escopo pois, como dito anteriormente, os créditos gerados neste caso pertencerão ao setor Energia porque este trabalho não analisa o tratamento do lodo.

*Para estes casos não se propões uma mudança de tecnologia, mas o aprimoramento, otimização de processos e manutenção a fim de alcançar máxima eficiência do sistema.

Tabela 3. Resultados do cálculo de RCEs e seu respectivo valor monetário. 
Por isso, recomendou-se apenas melhora dos procedimentos de revisão, monitoramento de indicadores e elevação dos níveis de eficiência. Todavia, este caso também não caracteriza adição ou substituição de tecnologia de projetos de MDL, mas sim boas práticas de manutenção, e assim não é pertinente o cálculo das RCEs.

As duas últimas situações discutidas não se enquadram nos princípios do mecanismo e, portanto dificilmente serão capazes de gerar RCEs. Desta forma, acredita-se que o potencial de obtenção de créditos de carbono do setor de tratamento de esgotos concentra-se na substituição de sistemas anaeróbios por aeróbios (alternativa “A”). Uma medida interessante e pouco explorada no Brasil tem sido praticada nos países desenvolvidos. Trata-se do uso da energia resultante de processos anaeróbios (queima do biogás) para suprir a demanda energética do metabolismo aeróbio, tornando sustentáveis as associações anaeróbio-aeróbio.

Além disso, é importante salientar que a proposição das alternativas de mitigação foi feita com base em apenas um parâmetro: a emissão de GEE. Todavia, existem outros critérios como área, custo e consumo energético que influenciam na tomada de decisão sobre o processo de tratamento de esgoto a ser implantado em cada caso.

\section{Conclusões}

O tratamento de esgotos sanitários é imprescindível na busca do desenvolvimento sustentável e o MDL pode permitir a viabilização econômica de infraestruturas e instalações para o saneamento por meio de créditos de carbono. Portanto, os gestores de recursos hídricos devem atentar e promover projetos na área.

Sob essa perspectiva este estudo buscou analisar esse potencial através da estimativa de geração de GEE nos processos de tratamento mais usuais e também de créditos de carbono. Os resultados mostraram que sistemas anaeróbios e abertos (lagoas e tanques sépticos) são maiores emissores de GEE e consequentemente tem maior relevância no contexto dos créditos de carbono. Em se tratando de sistemas fechados (reatores anaeróbios) existem tecnologias de recuperação e queima de gases, como flares, que minimizam esse dano, já frequentemente adotadas no Brasil. Por isso, verificou-se que o potencial inexplorado de obtenção de RCEs para a situação considerada no presente trabalho (sem $\mathrm{N}_{2} \mathrm{O}$ ), está na substituição de sistemas anaeróbios pelos aeróbios, cuja emissão de gases estufa, no caso, é nula.

A análise deste aspecto deve cada vez mais influenciar na escolha de alternativas no contexto urbano. Assim, é necessário que as cidades adotem uma postura pró-ativa por meio da prevenção do agravamento do aquecimento global (por exemplo, garantindo o bom funcionamento de ETEs) e do planejamento da mitigação do dano ao meio e à qualidade de vida.

\section{REFERÊNCIAS}

ABNT-ASSOCIAÇÃO BRASILEIRA DE NORMAS TÉCNICAS. NBR 12209/92: Projeto de estações de tratamento de esgoto sanitário. Rio de Janeiro, 1992.

CAKIRA, F. Y.; STENSTROM, M. K. Greenhouse gas production: A comparison between aerobic and anaerobic wastewater treatment technology. Water Research, n. 39, p. $4197-$ 4203, 2005.

CHERNICHARO, C. A. L. Reatores anaeróbios. 2. ed. Belo Horizonte: Departamento de Engenharia Sanitária e Ambiental, Universidade Federal de Minas Gerais, 2005. (Princípios do Tratamento de Biológico de Águas Residuárias, v. 05).

CETESB - COMPANHIA AMBIENTAL DO ESTADO DE SÃO PAULO. Inventário de Referência dos Gases de Efeito Estufa no Setor de Resíduos e Efluentes do Estado de São Paulo - Versão para consulta pública Out/2010. São Paulo: CETESB, 2010.

FADEL, M. EL.; MASSOUD, M. Methane emissions from wastewater management. Environment Pollution, n. 114, p. 177-185, 2001.

FGV-FUNDAÇÃO GETÚLIO VARGAS. O Mecanismo de Desenvolvimento Limpo - MDL: guia de orientação. Rio de Janeiro: Fundação Getulio Vargas, 2002.

IPCC-INTERNATIONAL PANEL ON CLIMATE CHANGE. The revised guidelines for national Greenhouse Gas Inventories: Reference manual, v. II e III. United Nations, New York, 1996. 
IPCC-INTERNATIONAL PANEL ON CLIMATE CHANGE. Good Practice Guidance and Uncertainty Management in National Greenhouse Gas Inventories. Japan: IPCC, 2000.

IPCC-INTERNATIONAL PANEL ON CLIMATE CHANGE. Climate Change 2001: The Scientific Basis. Contribution of Working Group I to the Third Assessment Report of the Intergovernmental Panel on Climate Change [Houghton, J.T.,Y. Ding, D.J. Griggs, M. Noguer, P.J. van der Linden, X. Dai, K. Maskell, and C.A. Johnson (eds.)]. Cambridge University Press, Cambridge, United Kingdom and New York, NY, USA, 2001.

IPCC-INTERNATIONAL PANEL ON CLIMATE CHANGE. 2006 IPCC Guidelines for National Greenhouse Gas Inventories. Japan: IPCC, 2006.

IPCC-INTERNATIONAL PANEL ON CLIMATE CHANGE. Climate Change 2007: Synthesis Report. Contribution of Working Groups I, II and III to the Fourth Assessment Report of the Intergovernmental Panel on Climate Change [Core Writing Team, Pachauri, R.K and Reisinger, A. (eds.)]. IPCC, Geneva, Switzerland, 2007.

KAMPSCHREUR, M. J. et al. Nitrous oxide emission during wastewater treatment. Water Research, Delft, v. 43, p. 4093-4103, 2009.

LUCAS, P. L. et al. Long-term reduction potential of non- $\mathrm{CO} 2$ greenhouse gases. Environmental science \& policy, n. 10, p. 85-103, 2007.

METCALF \& EDDY. Wastewater engineering: treatment and reuse. McGraw-Hill Book Co. 4 ed., 2003.

MCT-MINISTÉRIO DA CIÊNCIA E TECNOLOGIA. Protocolo de Quioto. Tradução: MCT, 1997.

MCT-MINISTÉRIO DA CIÊNCIA E TECNOLOGIA, 2009a. Inventário Brasileiro de emissões e remoções antrópicas de gases de efeito estufa: informações gerais e valores preliminares. Edição: MCT, 2009. Disponível em: <www. mct.gov.br>. Acesso em: 22 jul. 2010.

MCT-MINISTÉRIO DA CIÊNCIA E TECNOLOGIA, 2009b. MDL Ilustrado. IGES, Japão, 2009.
Disponível em: <www.mct.gov.br>. Acesso em: 21 jul. 2010.

MC-MINISTÉRIO DAS CIDADES. Sistema Nacional de Informações sobre Saneamento: diagnóstico dos serviços de água e esgotos 2008. Brasília: MCIDADES. SNSA, 2010.

ROSSO, D.; STENSTROM, M. K. The carbon-sequestration potential of municipal wastewater treatment. Chemosphere, n. 70, p. 1468 $-1475,2008$.

SALVADOR, N. N. B. Alternativas de Tratamento de Esgotos Sanitários (Notas de aula). DECiv/UFSCar, São Carlos, 2010.

SHAHABADI, M. B. et al. Estimation of greenhouse gas generation in wastewater treatment plants - Model development and application. Chemosphere, n. 78, p. 1085 1092, 2010.

USEPA-UNITED STATES ENVIRONMENTAL PROTECTION AGENCY. Inventory of U.S. Greenhouse Gas Emissions and Sinks: 1990 2008. U.S. Environmental Protection Agency, N.W. Washington, 2010.

VON SPERLING, M. Princípios básicos do tratamento de esgotos. Princípios do Tratamento Biológico de Águas Residuárias, v. 02. Belo Horizonte: Departamento de Engenharia Sanitária e Ambiental, Universidade Federal de Minas Gerais, 1996.

SOBRE OS AUTORES

Aline Pereira Lima*

Engenheira Ambiental pela Faculdade de Ciências e Tecnologia - FCT - UNESP, Campus de Presidente Prudente. Mestre em Engenharia Urbana pela Universidade Federal de São Carlos - UFSCar, Campus São Carlos.

Nemésio Neves Batista Salvador

Engenheiro Civil pela Universidade de Brasília - UnB. Mestre e Doutor em Hidráulica e Saneamento pela Escola de Engenharia de São Carlos - EESC - USP. Pós-doutor pela Oxford Brookes University. Professor do Programa de Pós- Graduação em Engenharia Urbana da Universidade Federal de São Carlos - UFSCar, Campus São Carlos.

*Endereço: Rua Manoel Eugênio, 633, Cidade Universitária; Presidente Prudente, SP, Brasil. CEP:19050-300.

E-mail: aline_lih@hotmail.com 


\section{E você achando que $100 \%$ fosse o máximo que alguém pudesse se dedicar.}

Sabesp 300\%: $100 \%$ de água tratada, $100 \%$ de esgoto coletado e $100 \%$ de esgoto tratado.

0 saneamento transforma a vida das pessoas. Por isso, não medimos esforços para levar, até 2014, 0 Sabesp 300\% para todos os municípios do interior atendidos pela Sabesp. E, até 2018, para todas as outras cidades atendidas por nós. Saiba mais acessando www.sabesp.com.br.

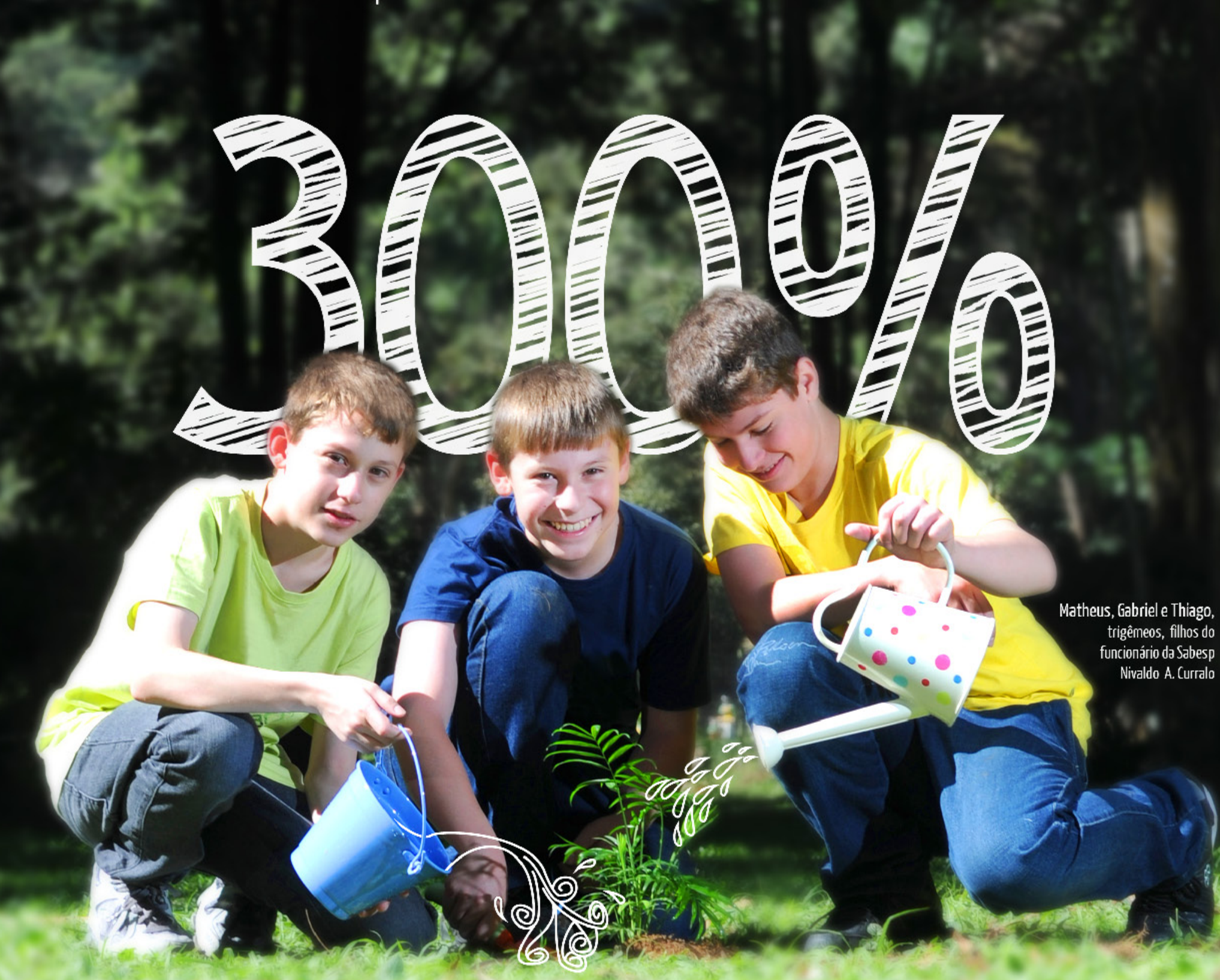

Sabesp.

Transformando vidas. 